\section{E-044 STENTING OF INTRACRANIAL STENOSIS THROUGH AN ANGIOPLASTY BALLOON CATHETER - A CASE SERIES}

${ }^{1} \mathrm{~A}$ Takayanagi ${ }^{*},{ }^{2} \mathrm{~J}$ Fields, ${ }^{2} \mathrm{~K}$ Chao, ${ }^{2} \mathrm{D}$ Rex, ${ }^{2} \mathrm{P}$ Cheng, ${ }^{2} \mathrm{~L}$ Feng. ${ }^{1}$ Riverside University Health Systems, Moreno Valley, CA; ${ }^{2}$ Kaiser Permanente Los Angeles Medical Center, Los Angeles, CA

\subsection{6/neurintsurg-2021-SNIS. 140}

Introduction Microcatheter exchange increases the risks of intracranial angioplasty and stenting using the Wingspan/Gateway system and may have contributed to the high procedural risks in the SAMMPRIS trial. We present a case series of direct stent deployment through the balloon catheter, simplifying the technique of intracranial angioplasty and stenting using self-expanding stents.

Materials and Methods We retrospectively reviewed all patients who underwent stenting for intracranial stenosis with deployment of the Neuroform Atlas stent through the Gateway balloon in our hospital system since this technique was first utilized in October 2020. Procedural success, complication rate, short term clinical and imaging follow-up were assessed.

Results Ten Neuroform Atlas stents were deployed through either the Gateway or Emerge balloon catheter in eight patients. The median age was 59 (range 30-75) and 50\% were female. All patients had symptomatic severe intracranial stenosis with mean stenosis of $83 \%$ (range $70 \%$ to $90 \%$ ). Six patients had recurrent strokes and had failed medical therapy, while two patients presented with acute stroke symptoms and were treated emergently. Mean post-stenting stenosis was 32\% (range $0 \%$ to $50 \%$ ). Two procedures were performed under conscious sedation and the rest under general anesthesia. Four stents were placed in the supraclinoid internal carotid artery (ICA), one in the middle cerebral artery M1, two in M2, two in the basilar artery and one in the intracranial vertebral artery. All five patients who underwent short-term follow-up angiograms (mean interval 1.2 months) had further improvement of residual stenosis. There were no ischemic or

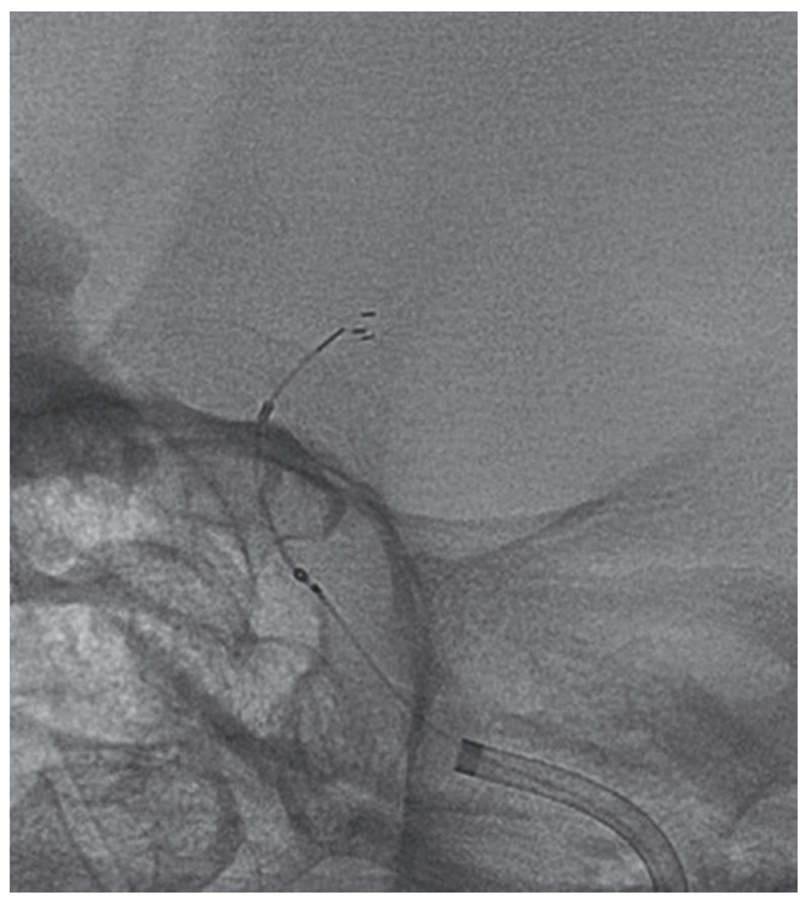

Abstract E-044 Figure 1 hemorrhagic periprocedural complications. None of the patients had recurrent TIAs or strokes. One patient died of a COVID-19 infection. One patient had transient neurological deterioration at four months due to profound hypoglycemia.

Conclusion Deploying self-expanding stents through an angioplasty balloon catheter is feasible, safe, and can greatly simplify the procedure. The Neuroform Atlas stent is strong enough to prevent immediate recoil of intracranial stenosis after angioplasty.

Disclosures A. Takayanagi: None. J. Fields: None. K. Chao: None. D. Rex: None. P. Cheng: None. L. Feng: None.

\section{E-045 EARLY NEUROLOGICAL IMPROVEMENT IS INDEPENDENT PREDICTOR OF FAVORABLE OUTCOME FOLLOWING THROMBECTOMY FOR BASILAR ARTERY OCCLUSION}

S Majidi*, S Matsoukas, R De Leacy, T Oxley, H Shoirah, T Shigematsu, C Kellner, J Mocco, J Fifi. Neurosurgery, Mount Sinai Hospital, New York, NY

\subsection{6/neurintsurg-2021-SNIS.141}

Background Acute ischemic stroke from basilar artery occlusion (BAO) is a devastating disease with high mortality and morbidity rate. The safety and efficacy of endovascular thrombectomy in BAO is not fully understood. We aimed to evaluate the predictors of favorable clinical outcome in these patients.

Methods We performed a retrospective analysis of consecutive acute stroke patients with $\mathrm{BAO}$ whom underwent endovascular thrombectomy. Demographics, and the rate of successful recanalization (TICI score $\geq 2 \mathrm{~B}$ ) were reviewed. The primary efficacy outcome was defined as 90-day modified Rankin Scale (mRS) score $\leq 2$ (or no worsening of $\mathrm{mRS}$ at 90 days compared to pre-stroke mRS in patients with pre-stroke $m R S>2$ ). Secondary outcome included early neurological improvement (ENI) defined as $\geq 8$ points reduction in National Institutes of Health Stroke Scale (NIHSS) score, as compared to presentation, or NIHSS score $\leq 4$ at 24 hours. Multivariate analysis was performed to determine predictors of favorable 90-day outcome.

Results Sixty-two patients were identified with mean (SD) age 67 (15), 39\% women, and 24\% African-American. Initial NIHSS median (IQR) was 22 (14), the mean symptoms onset to groin puncture time was 9.7 (5.7) hours. Thirty-one percent of patients were treated with intravenous thrombolytic therapy. Successful recanalization was achieved in $92 \%$ of the patients. Total of $34 \%$ of the patients had favorable 90 -day outcome. Mortality at 90-day was seen in $34 \%$ of the patients. ENI was observed in 22 (35\%) patients. Patients with ENI were more likely to have favorable 90-day clinical outcome, compared to those without ENI (52\% vs $27 \%$, $\mathrm{p}=0.04)$. Single pass successful recanalization (OR; 95\% CI: $5.56 ; 1.61-19.81, \mathrm{p}=0.005)$ and initial NIHSS score (OR; 95\% CI: $1.11 ; 1.2-1.19, \mathrm{p}=0.043)$ were independent predictors of ENI.

Conclusion ENI is an independent predictor of favorable clinical outcome after BAO thrombectomy. Single pass successful recanalization is associated with higher likelihood of ENI.

Disclosures S. Majidi: 1; C; SNIS Foundation. S. Matsoukas: None. R. De Leacy: None. T. Oxley: None. H. Shoirah: None. T. Shigematsu: None. C. Kellner: None. J. Mocco: None. J. Fifi: None. 